\title{
Сингулярность плотности состояний и анизотропия транспорта в двумерном электронном газе со спин-орбитальным взаимодействием в параллельном магнитном поле
}

\author{
(C) В.А. Сабликов, Ю.Я. Ткачฯ \\ Институт радиотехники и электроники им. В.А. Котельникова Российской академии наук \\ (Фрязинский фрилиал), \\ 141190 Фрязино, Россия \\ ฯ E-mail: utkach@gmail.com
}

(Получена 25 апреля 2018 г. Принята к печати 7 мая 2018 г.)

\begin{abstract}
Двумерный электронный газ со спин-орбитальным взаимодействием в параллельном магнитном поле, как известно, образует анизотропную систему с сингулярностью Ван Хова плотности состояний, управляемую магнитным полем. Мы исследовали тензоры проводимости и спиновой восприимчивости, определяющей эффект Эдельштейна, для этой системы и установили, что проводимость и спиновая восприимчивость имеют резкие особенности при изменении магнитного поля или положения уровня Ферми, возникающие при прохождении уровня Ферми через точку сингулярности.
\end{abstract}

DOI: $10.21883 /$ FTP.2018.12.46760.40

\section{1. Введение}

Низкоразмерные электронные системы со спин-орбитальным взаимодействием (СОВ) вызывают исключительно большой интерес, связанный с множеством качественно новых физических эффектов [1,2], многие из которых имеют и большое практическое значение [3-5]. Наибольшее внимание привлекают двумерные (2D) системы, в которых СОВ может управляться внешним электрическим полем [2]. Настоящая работа посвящена эффектам, возникающим в 2D электронном газе (ЭГ) c СОВ при наличии параллельного магнитного поля, которое, очевидно, приводит к анизотропии электронного спектра и соответственно к анизотропии транспорта. Интерес к такого рода анизотропной системе вызван двумя причинами. Во-первых, она моделирует анизотропию электронной системы с СОВ, возникающую вследствие сильного электрон-электронного взаимодействия без внешнего магнитного поля [6], но в отличие от нее допускает относительно простое решение. Во-вторых, в последнее время транспорт в параллельном магнитном поле становится важным инструментом экспериментального исследования 2D электронных систем с сильным СОВ.

Кроме того двумерный электронный газ в условиях сильного СОВ еще недостаточно исследован, и в последнее время обнаруживаются новые особенности. Так, недавние теоретические исследования проводимости 2D ЭГ с СОВ показали, что проводимость $(G)$ имеет необычную зависимость от плотности электронов $n$, когда она мала так, что заполнена лишь нижняя спиновая подзона, а при большой плотности приобретает обычный друдевский вид [7]:

$$
G=\frac{e^{2} n \tau_{0}}{2 m} \begin{cases}\frac{n^{3}}{n_{0}^{3}}+\frac{n}{n_{0}}, & n \leq n_{0}, \\ 2, & n \geq n_{0},\end{cases}
$$

где $\tau_{0}$ - характерное время рассеяния в отсутствие $\mathrm{COB}, m$ - эффективная масса, $n_{0}=E_{\text {so }} /\left(\pi \hbar^{2}\right)-$ концентрация на уровне Ферми, когда он совпадает с дираковской точкой, ниже которой имеется только одна спиновая подзона; $E_{s o}=\alpha^{2} m /\left(2 \hbar^{2}\right)-$ характерная энергия СОВ, $\alpha-$ постоянная СОВ Рашбы.

Расчеты спиновой поляризации, возникающей при наличии электрического поля вдоль 2D слоя (эффект Эдельштейна $[8]$ ), показывают, что спиновая поляризация растет пропорционально энергии Ферми ниже дираковской точки и достигает насыщения выше еe [9].

При наличии параллельного магнитного поля проводимость и спиновая поляризация 2D ЭГ с СОВ практически не исследовались (особенно в области малых концентраций, $n<n_{0}$ ), хотя в этом случае можно ожидать появления нетривиальных особенностей, связанных с сингулярностью плотности состояний Ван Хова, которая, как мы недавно установили [10], имеется при энергии ниже дираковской точки. Сингулярность имеет логарифмический характер. Она образуется благодаря наличию магнитного поля из корневой сингулярности плотности состояний, существующей в минимуме энергии 2D ЭГ с СОВ при нулевом магнитном поле, $N(E) \propto\left(E+E_{s o}\right)^{-1 / 2}$. С ростом магнитного поля точка сингулярности, отвечающая седловой точке в $k$-пространстве, смещается от дна зоны до точки Дирака, а после этого исчезает. Эта интересная особенность открывает новые возможности экспериментального исследования спектра систем с сильным СОВ путем сканирования магнитного поля и измерения транспортных характеристик.

В настоящей работе приведены результаты исследования проводимости и спиновой поляризации в эффекте Эдельштейна для 2D ЭГ с СОВ Рашбы в параллельном магнитном поле. 


\section{2. Постановка задачи и методы}

Гамильтониан 2D ЭГ с СОВ Рашбы в магнитном поле В, направленном вдоль оси $x$, имеет вид

$$
H=\frac{p^{2}}{2 m} \sigma_{0}+\frac{\alpha}{\hbar}\left(p_{x} \sigma_{y}-p_{y} \sigma_{x}\right)-b_{1} \sigma_{x}
$$

где $\mathbf{p}=\left(p_{x}, p_{y}\right)-$ импульс электрона, $\sigma_{x}$ и $\sigma_{y}-$ матрицы Паули, $b_{1}=\left(g^{*} / 2\right) \mu_{\mathrm{B}} B, g^{*}-$ эффективный $g$-фактор, который предполагается изотропным и не зависящим от величины магнитного поля, $\mu_{\mathrm{B}}-$ магнетон Бора. Векторный потенциал выбран в калибровке $\mathbf{A}=(0,0, y B)$, при которой импульс совпадает с обобщенным импульсом.

Закон дисперсии и собственные волновые функции для этого гамильтониана в безразмерных обозначениях имеют вид

$$
\begin{gathered}
E_{\lambda}(\mathbf{k})=k^{2}+2 \lambda \sqrt{k^{2}+2 k b \sin \phi+b^{2}}, \\
\Psi_{\lambda}(\mathbf{k})=\frac{1}{\sqrt{2 A}}\left(\begin{array}{c}
1 \\
i \lambda e^{i \varphi}
\end{array}\right) e^{i\left(k_{x} x+k_{y} y\right)},
\end{gathered}
$$

где волновой вектор $\mathbf{k}=k(\cos \phi, \sin \phi)$ нормирован на $k_{s o}=\alpha m / \hbar^{2}$, энергия нормирована на $E_{s o}$ и введено безразмерное магнитное поле $b=\left(g^{*} / 2\right) \mu_{\mathrm{B}} B / \alpha k_{s o}$, $\lambda= \pm 1$ - спиновый индекс, $A$ - площадь образца. Угол $\varphi$ определяет ориентацию спина, он связан с $\phi$ соотношением:

$$
\tan \varphi=(k \sin \phi+b) /(k \cos \phi) .
$$

Для нахождения проводимости и поляризации в условиях протекания тока необходимо вычислить функцию распределения $f_{\lambda}(\mathbf{k}, \varepsilon)$. Для этого мы воспользуемся квазиклассическим кинетическим уравнением Больцмана [11]:

$$
\begin{aligned}
-e \mathscr{E} \mathbf{v}_{\lambda}(\mathbf{k})\left(-\partial_{E} f_{0}\right)=\sum_{\lambda^{\prime}} & \int \frac{d^{2} k^{\prime}}{(2 \pi)^{2}} W_{\lambda \lambda^{\prime}}\left(\mathbf{k}, \mathbf{k}^{\prime}\right) \\
\times & {\left[f_{\lambda}(\mathbf{k}, \mathscr{E})-f_{\lambda^{\prime}}\left(\mathbf{k}^{\prime}, \mathscr{E}\right)\right], }
\end{aligned}
$$

где $\mathscr{E}-$ напряженность электрического поля, направленного под углом $\theta$ к оси $x, \mathscr{E}=\mathscr{E}(\cos \theta, \sin \theta)$, величина $\mathscr{E}$ нормирована на $E_{s o} k_{s o} / e, \mathbf{v}_{\lambda}(\mathbf{k})-$ групповая скорость, $f_{0}$ - равновесная функция распределения.

Для простоты рассмотрим случай, когда рассеяние происходит на примесях с короткодействующим потенциалом $V(\mathbf{r})=V_{0} \delta(\mathbf{r})$ и является упругим. Это предположение не является принципиальным для качественной оценки поведения вычисляемых нами откликов. Вероятность электронных переходов в борновском приближении имеет вид

$$
W_{\lambda \lambda^{\prime}}\left(\mathbf{k}, \mathbf{k}^{\prime}\right)=R\left|\left\langle\mathbf{k}^{\prime}, \lambda^{\prime}\left|V / V_{0}\right| \mathbf{k}, \lambda\right\rangle\right|^{2} \delta\left(E_{\mathbf{k}, \lambda}-E_{\mathbf{k}^{\prime}, \lambda^{\prime}}\right),
$$

где $R=n_{i} V_{0}^{2} k_{s o}^{2} /\left(2 \pi E_{s o}^{2}\right), n_{i}$ - концентрация рассеивающих примесей.
При интегрировании по k в уравнении (5) необходимо иметь в виду, что при любой энергии существуют два контура Ферми в k-пространстве, как выше дираковской точки, так и ниже точки сингулярности. В первом случае два контура относятся к состояниям с разным спиновым индексом, а во втором имеются два замкнутых контура для состояний с $\lambda=-1$. В интервале энергии выше точки сингулярности и ниже дираковской точки имеется один односвязный контур, но такой, что имеются два значения $\mathbf{k}$ в определенном интервале $E$. Отметим, что из (3) следует: дираковская точка имеет координаты $(0, b)$ в $\mathbf{k}$-пространстве и отвечает энергии $b^{2}$. Более подробно изменения контура Ферми в магнитном поле приведены в работе [10].

Важной особенностью рассматриваемой задачи является тот факт, что в условиях анизотропии интеграл столкновений не может быть упрощен путем введения эффективного времени рассеяния [12]. Даже использование так называемого двухвременно́го приближения $[13,14]$ приводит к ошибочным результатом. Следуя работе [12], мы решаем кинетическое уравнение (5) точно, без использования приближения времени релаксации.

С учетом упругости рассеяния решение уравнения (5) на контурах Ферми представим в виде

$$
\begin{aligned}
\Delta f_{\lambda} & =f_{\lambda}(\phi, \theta)-f_{0} \\
& =e \mathscr{E} \partial_{E} f_{0} \omega_{\lambda}(\phi)\left[a_{\lambda}(\phi) \cos \theta+b_{\lambda}(\phi) \sin \theta\right],
\end{aligned}
$$

где $\omega_{\lambda}(\phi)$ - величина групповой скорости на соответствующем контуре $\omega_{\lambda}(\mathbf{k})=\omega_{\lambda}(\phi)\left(\cos \xi_{\lambda}, \sin \xi_{\lambda}\right)$, а $\xi_{\lambda}(\phi)$ - угол, определяющий ее направление.

Наличие $\delta$-функции в уравнении (6) позволяет провести интегрирование по величине $k$ в уравнении (5), так что в нем остаются интегралы только по углу $\phi$ на соответствующих контурах Ферми. В результате получим две несвязанные системы уравнений для величин $a_{\lambda}$ и $b_{\lambda}$ :

$$
\left\{\begin{aligned}
\cos \xi_{ \pm}=\bar{W}_{ \pm}\left(a_{ \pm}\right)(\phi) & -\int d \phi^{\prime}\left[\frac{v_{ \pm}\left(\phi^{\prime}\right)}{v_{ \pm}(\phi)} W_{ \pm \pm}\left(\phi, \phi^{\prime}\right) a_{ \pm}\left(\phi^{\prime}\right)\right. \\
& \left.+\frac{v_{ \pm}\left(\phi^{\prime}\right)}{v_{ \pm}(\phi)} W_{ \pm \mp}\left(\phi, \phi^{\prime}\right) a_{\mp}(\phi)\right] \\
\sin \xi_{ \pm}=\bar{W}_{ \pm}\left(b_{ \pm}\right)(\phi) & -\int d \phi^{\prime}\left[\frac{v_{ \pm}\left(\phi^{\prime}\right)}{v_{ \pm}(\phi)} W_{ \pm \pm}\left(\phi, \phi^{\prime}\right) b_{ \pm}\left(\phi^{\prime}\right)\right. \\
& \left.+\frac{v_{ \pm}\left(\phi^{\prime}\right)}{v_{ \pm}(\phi)} W_{ \pm \mp}\left(\phi, \phi^{\prime}\right) b_{\mp}\left(\phi^{\prime}\right)\right]
\end{aligned}\right.
$$

Здесь

$$
\begin{aligned}
W_{\lambda, \lambda^{\prime}}\left(\phi, \phi^{\prime}\right) & =\int_{0}^{\infty} d k^{\prime} k W_{\lambda \mathbf{k}, \lambda^{\prime} \mathbf{k}^{\prime}} \\
= & \frac{R}{2}\left[\frac{k^{\prime 2}\left\{1+\lambda \lambda^{\prime} \cos \left[\varphi_{\lambda}(\phi)-\varphi_{\lambda^{\prime}}\left(\phi^{\prime}\right)\right]\right\}}{\left|\mathbf{k}^{\prime} \nabla_{\mathbf{k}^{\prime}} E_{\lambda}\left(\mathbf{k}^{\prime}\right)\right|}\right]_{k^{\prime}=k_{\lambda^{\prime}}(\phi)}
\end{aligned}
$$


и

$$
\bar{W}_{\lambda}=\sum_{\lambda^{\prime}} \int d \phi^{\prime} W_{\lambda, \lambda^{\prime}}\left(\phi, \phi^{\prime}\right)=\sum_{\lambda^{\prime}} W_{\lambda^{\prime}},
$$

где угол ориентации спина $\varphi_{\lambda}(\phi)$ определяется соотношением

$$
\tan \varphi_{\lambda}(\phi)=\frac{k_{\lambda}(\phi) \sin \phi+b}{k_{\lambda}(\phi) \cos \phi},
$$

вычисляемым на контуре Ферми.

Уравнения (8) решаются точно. В настоящей работе мы приведем результаты численного решения, полученного на сетке по углу $\phi$ для каждого из контуров. Нетривиальным моментом является то обстоятельство, что детерминант образующейся системы линейных уравнений равен нулю и ранг расширенной матрицы системы равен рангу детерминанта, причем этот ранг на единицу меньше размерности угловой сетки. Это означает, что для решения системы необходимо использовать дополнительное уравнение. Очевидно, что такое уравнение необходимо получить из условия сохранения числа частиц, которое в данном случае совпадает с условием электронейтральности системы.

Сначала показательно рассмотреть случай, когда магнитное поле отсутствует, и получить согласие полученных по предложенной здесь методике результатов с известными в литературе. При нулевом магнитном поле система уравнений (8) существенно упрощается, так как групповая скорость $v_{ \pm}=$const в силу аксиальной симметрии контуров Ферми. В результате приходим к следующему выражению для функции распределения:

$$
\begin{aligned}
\nabla f_{ \pm}(\phi, 0)= & e v_{ \pm} \mathscr{E} \partial_{E} f_{0} \frac{2 W_{ \pm}}{\left(W_{+}+W_{-}\right)^{2}} \\
& \times \cos (\theta-\phi) \begin{cases} \pm 1 & \text { при } E_{\mathrm{F}}<0 \\
1 & \text { при } E_{\mathrm{F}}>0\end{cases}
\end{aligned}
$$

Здесь коэффициенты $W_{ \pm}$зависят только от положения уровня Ферми (или концентрации электронов):

$$
W_{ \pm}=\frac{1}{2 \tau_{0}} \begin{cases}\frac{1 \pm \sqrt{E_{\mathrm{F}}+1}}{\sqrt{E_{\mathrm{F}}+1}}, & -1 \leq E_{\mathrm{F}} \leq 0, \\ \frac{\sqrt{E_{\mathrm{F}}+1} \pm 1}{\sqrt{E_{\mathrm{F}}+1}}, & E_{\mathrm{F}}>0,\end{cases}
$$

где $\tau_{0}=\hbar / R E_{s o}$.

Проводимость, вычисленная с помощью выражения (12) для неравновесной функции распределения, совпадает с результатом, полученным ранее в работе [7] с использованием формулы Кубо и представленным уравнением (1). Спиновая поляризация также согласуется с полученной в работе [8].

Новым результатом, к которому приводит использованный нами подход, является корректное определение времени рассеяния с помощью соотношений (12), (13). Мы установили, что система описывается двумя временами релаксации, $\tau_{+}$и $\tau_{-}$, относящимися к разным контурам поверхности Ферми: $\tau_{ \pm}=2 W_{ \pm} /\left(W_{+}+W_{-}\right)^{2}$. Они

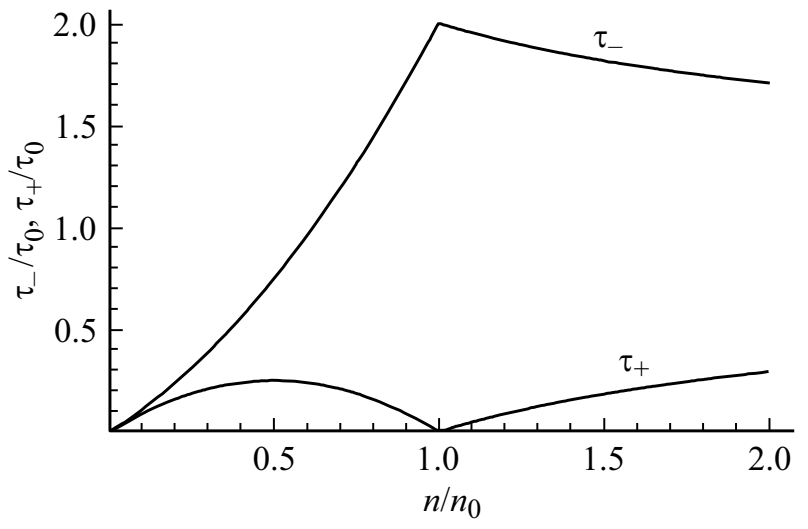

Рис. 1. Времена рассеяния $\tau_{+}$и $\tau_{-}$для двух контуров Ферми при нулевом магнитном поле в зависимости от концентрации электронов $n$. Индексы \pm соответствуют спиновым индексам $\lambda= \pm 1$ при $n>n_{0}$. В области $n<n_{0}$ индексы \pm соответствуют двум контурам Ферми с $\lambda=-1$.

существенно различаются по величине и, как показано на рис. 1, имеют разную зависимость от концентрации электронов.

\section{3. Основные результаты}

Компоненты тензора проводимости выражаются через функции $a_{\lambda}(\phi)$ и $b_{\lambda}(\phi)$ следующим образом:

$$
\begin{aligned}
G_{x x} & =\sum_{\lambda} \int d \phi F_{\lambda}(\phi) a_{\lambda}(\phi) \cos \xi_{\lambda}(\phi), \\
G_{x y} & =\sum_{\lambda} \int d \phi F_{\lambda}(\phi) b_{\lambda}(\phi) \cos \xi_{\lambda}(\phi), \\
G_{y x} & =\sum_{\lambda} \int d \phi F_{\lambda}(\phi) a_{\lambda}(\phi) \sin \xi_{\lambda}(\phi), \\
G_{y y} & =\sum_{\lambda} \int d \phi F_{\lambda}(\phi) b_{\lambda}(\phi) \sin \xi_{\lambda}(\phi),
\end{aligned}
$$

где

$$
F_{\lambda}(\phi)=\frac{v_{\lambda}^{2}(\phi) k_{\lambda}(\phi)}{\left|\left[\partial_{k} E_{\lambda}(k, \phi)\right]\right|_{k=k_{\lambda}(\phi)}} .
$$

Здесь проводимость нормирована на величину $G_{0}=2 e^{2} / h R$.

Из соображений симметрии контуров Ферми нетрудно показать, что недиагональные элементы тензора проводимости равны нулю $\left(G_{x y}=G_{y x}=0\right)$. Диагональные же компоненты имеют нетривиальную зависимость от магнитного поля. Она характеризуется наличием резкого минимума проводимости при определенном значении магнитного поля. Он возникает из-за того, что при изменении магнитного поля изменяется положение уровня Ферми относительно точки сингулярности плотности состояний. Минимум достигается, когда уровень Ферми совпадает с точкой сингулярности. При этом происходит 


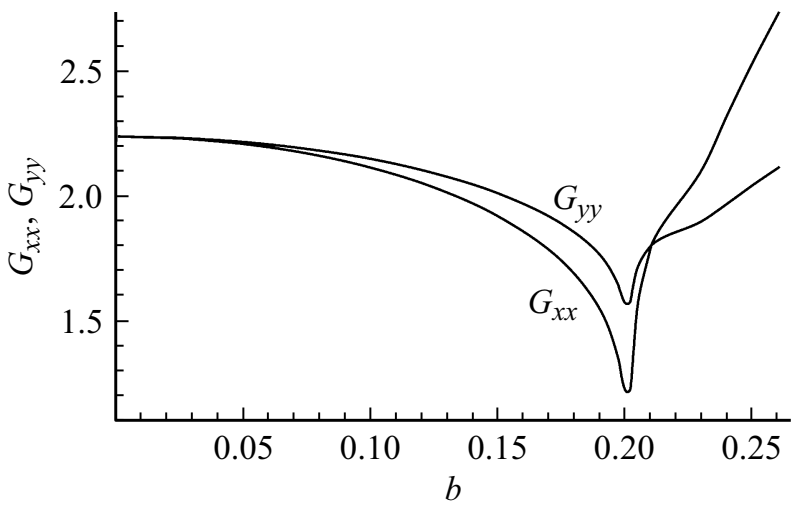

Рис. 2. Зависимости диагональных элементов тензора проводимости от магнитного поля в области энергии Ферми ниже дираковской точки. Расчеты проведены для энергии Ферми $E_{\mathrm{F}}=-0.6$.
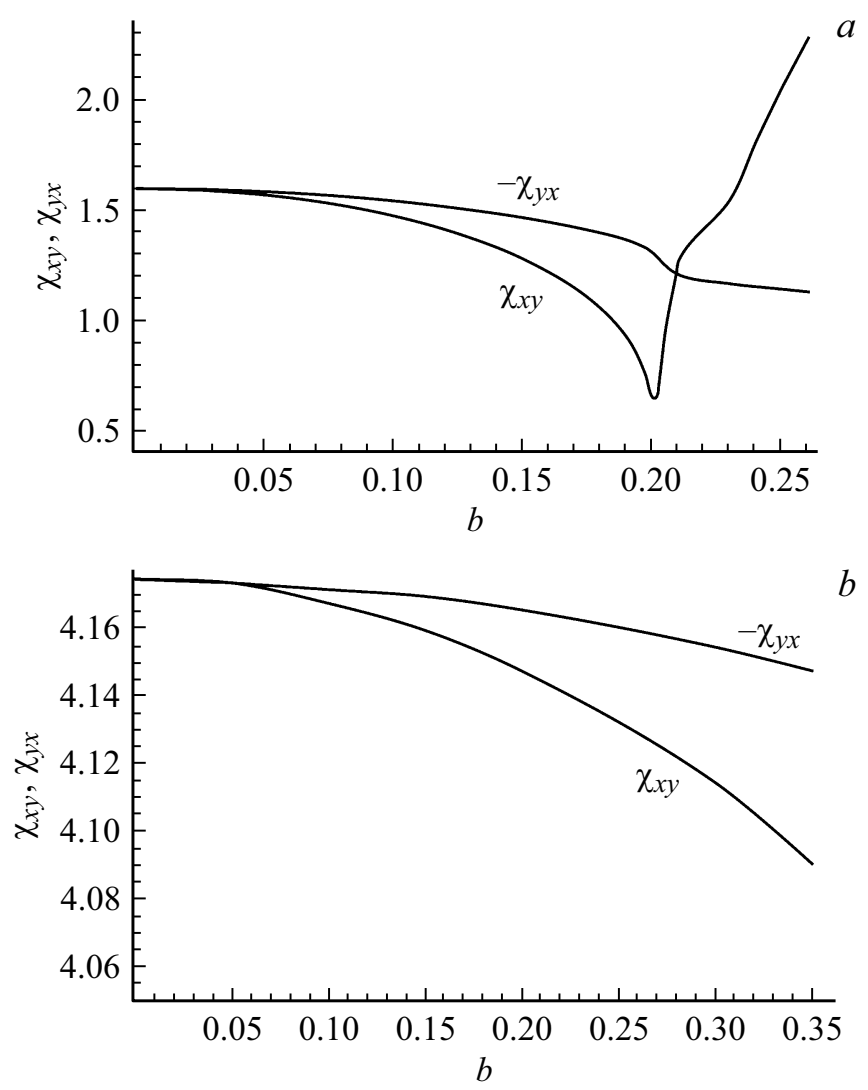

Рис. 3. Зависимости элементов тензора спиновой восприимчивости от магнитного поля для энергии Ферми ниже дираковской точки, $E_{\mathrm{F}}=-0.6(a)$, и выше дираковской точки, $E_{\mathrm{F}}=0.8(b)$.

наиболее сильное рассеяние электронов. Положение минимума проводимости определяется условием

$$
E_{\mathrm{F}}=-1+2 b \text {. }
$$

Зависимость диагональных компонент тензора проводимости от магнитного поля показана на рис. 2 для случая, когда энергия Ферми ниже дираковской точки. Если же энергия Ферми выше дираковской точки, то анизотропия проводимости отсутствует.

Рассмотрим теперь спиновую плотность $\mathbf{S}$, образующуюся при протекании тока. Компоненты плотности $S_{i}$ $(i=x, y, z)$ определяются следующим образом:

$$
S_{i}=\frac{\hbar}{2} \sum_{\lambda} \int \frac{d^{2} k}{4 \pi^{2}}\left\langle\Psi_{\lambda, k}^{+}\left|\sigma_{i}\right| \Psi_{\lambda, k}\right\rangle \nabla f_{\lambda}(\mathbf{k})
$$

Соответствующие восприимчивости $\chi_{i j}$ определяются уравнением

$$
S_{i}=\sum_{j} \chi_{i j} \mathscr{E}_{j}
$$

Используя уравнения (22) и (12), находим следующие выражения для спиновой восприимчивости:

$$
\begin{aligned}
& \chi_{x x}=\sum_{\lambda} \lambda \int d \phi S_{\lambda}(\phi) a_{\lambda}(\phi) \sin \varphi_{\lambda}(\phi), \\
& \chi_{x y}=\sum_{\lambda} \lambda \int d \phi S_{\lambda}(\phi) b_{\lambda}(\phi) \sin \varphi_{\lambda}(\phi), \\
& \chi_{y x}=\sum_{\lambda} \lambda \int d \phi S_{\lambda}(\phi) a_{\lambda}(\phi) \cos \varphi_{\lambda}(\phi), \\
& \chi_{y y}=\sum_{\lambda} \lambda \int d \phi S_{\lambda}(\phi) b_{\lambda}(\phi) \cos \varphi_{\lambda}(\phi) .
\end{aligned}
$$

Здесь все компоненты спиновой поляризации нормированы на $e \alpha m \tau_{0} / h$ и локальную плотность на контуре Ферми определяет множитель

$$
S_{\lambda}(\phi)=\frac{v_{\lambda}(\phi) k_{\lambda}(\phi)}{\left[\partial_{k} E_{\lambda}(k, \varphi)\right]_{k=k_{\lambda}(\phi)}} .
$$

Соображения симметрии показывают, что диагональные компоненты тензора $\chi_{i j}$ равны нулю и отсутствует нормальная компонента спиновой поляризации, $S_{z}=0$. Недиагональные компоненты $\chi_{i j}$ имеют зависимость от магнитного поля с характерной особенностью при выполнении условия (18). Компонента $\chi_{x y}$ имеет резкий минимум, а компонента $\chi_{y x}$ - точку перегиба. Поведение спиновой поляризации в зависимости от магнитного поля показано на рис. 3 для положений уровня Ферми ниже и выше дираковской точки.

\section{4. Заключение}

Мы показали, что под действием параллельного магнитного поля возникают две характерные особенности в поведении проводимости и спиновой восприимчивости 2D ЭГ с СОВ. Во-первых, образуется достаточно резкая особенность (минимум проводимости и восприимчивости $\chi_{x y}$ ) при энергии Ферми ниже дираковской точки. Она образуется при условии, когда уровень Ферми совпадает с точкой сингулярности Ван Хова. 
Экспериментальное наблюдение такой особенности может быть использовано для определения константы СОВ с помощью уравнения (18). Во-вторых, возникает анизотропия проводимости и спиновой восприимчивости, которая также может быть инструментом изучения электронных систем с СОВ. Заметим, однако, что найденные в этой работе закономерности получены для невзаимодействующих электронных систем. Пренебрежение взаимодействием оправдано при достаточно большой диэлектрической проницаемости и для систем с сильным СОВ, таким, что энергия $\mathrm{COB} E_{s o}$ значительно превосходит энергию кулоновского взаимодействия электронов. Такие условия могут быть выполнены для широко изучаемых в настоящее время электронных систем с гигантским СОВ на основе BiTeI $[15,16]$, границ раздела $\mathrm{LaAlO}_{3} / \mathrm{SrTiO}_{3}$ [17] и поверхностных сплавов $[18,19]$. В частности, оценка для $\mathrm{BiTeI}$, где $E_{s o} \approx 0.1$ эВ, $m \approx 0.2 m_{0}$ и диэлектрическая проницаемость $\varepsilon=15$ [15], приводит к концентрации $n_{0} \approx 1.9 \cdot 10^{13} \mathrm{~cm}^{-2}$ и энергии кулоновского взаимодействия $e^{2} \sqrt{n_{0}} / \varepsilon \approx 0.04$ эВ. Масштаб магнитных полей, при которых могут наблюдаться предсказанные нами сингулярности, оценивается из равенства $b=1$ и численно имеет порядок 10 Тл.

Работа выполнена при частичной поддержке РФФИ (проект № 17-02-00309) и Программы президиума РАН 1.4. „Актуальные проблемы физики низких темпеpaтур $^{6 .}$.

\section{Список литературы}

[1] A. Manchon, H.C. Koo, J. Nitta, S.M. Frolov, R.A. Duine. Nature Materials, 14, 871 (2015).

[2] R. Winkler. Spin-Orbit Coupling Effects in Two-Dimensional Electron and Hole Systems (Springer, 2003).

[3] J. Zutic, J. Fabian, S. Das Sarma. Rev. Mod. Phys., 76, 323 (2004).

[4] J Fabian, A. Matos-Abiague, C. Ertler, P. Stano, I. Zutic. Acta Physica Slovaca, 57, 565 (2007).

[5] D.D. Awschalom, M.J. Flatt'e. Natire Phys., 3, 153 (2007).

[6] E. Berg, M.S. Rudner, S.A. Kivelson. Phys. Rev. B, 85, 035116 (2012).

[7] V. Brosco, L. Benfatto, E. Cappulluti, C. Grimaldi. Phys. Rev. Lett., 116, 166602 (2016).

[8] V. Edelstein. Solid State Commun., 73, 233 (1990).

[9] A. Johansson, J. Henk, I. Mertig. Phys. Rev. B, 93, 195440 (2016).

[10] Ю.Я. Ткач. Письма ЖЭТФ, 104, 102 (2016).

[11] А. Абрикосов. Основы теории металлов (М., Наука, 1987).

[12] K. Vyborny, A.A. Kovalev, J. Sinova, T. Jungwirth. Phys. Rev. B, 79, 045427 (2009).

[13] J. Shliemann, D. Loss. Phys. Rev. B, 68, 165311 (2003).

[14] M. Trushin, J. Shliemann. Phys. Rev. B, 75, 155323 (2007).

[15] M. Sakano, M.S. Bahramy, A. Katayama, T. Shimojima, H. Murakawa, Y. Kaneko, W. Malaeb, S. Shin, K. Ono, H. Kumigashira, R. Arita, N. Nagaosa, H.Y. Hwang, Y. Tokura, K. Ishizaka. Phys. Rev. Lett., 110, 107204 (2013).
[16] F.-X. Xiang, X.-L. Wang, M. Veldhorst, S.X. Dou, M.S. Fuhrer. Phys. Rev. B, 92, 035123 (2015).

[17] S. Hurand, A. Jouan, C. Feuillet-Palma, G. Singh, J. Biscaras, E. Lesne, N. Reyren, A. Barthlmy, M. Bibes, J.E. Villegas, C. Ulysse, X. Lafosse, M. Pannetier-Lecoeur, S. Caprara, M. Grilli, J. Lesueur, N. Bergeal. Sci. Rep., 5, 12751 (2015).

[18] C.A. Ast, J. Henk, A. Ernst, L. Moreschini, M.S. Falub, D. Pacile, P. Bruno, K. Kern, M. Grioni. Phys. Rev. Lett., 98, 186807 (2007).

[19] I. Gierz, T. Suzuki, E. Frantzeskakis, S. Pons, S. Ostanin, A. Ernst, J. Henk, M. Grioni, K. Kern, C.R. Ast. Phys. Rev. Lett., 103, 046803 (2009).

Редактор Л.В. Шаронова

\section{Singularity of the density of states and transport anisotropy in two-dimensional electron gas with spin-orbit interaction in in-plane magnetic field}

\author{
V.A. Sablikov, Yu.Ya. Tkach \\ Kotel'nikov Institute of Radio Engineering and \\ Electronics (Fryazino Branch), \\ Russian Academy of Sciences, \\ 141190 Fryazino, Russia
}

\begin{abstract}
Two-dimensional electron gas with spin-orbit interaction in an in-plane magnetic field is known to form an anisotropic system with a van Hove singularity of the density of states controlled by the magnetic field. We study tensors of the conductivity and spin susceptibility when determine the Edelstein effect for this system. It was found that both the conductivity and the spin susceptibility have sharp singularities, which can be observed with varying the magnetic field or the position of the Fermi level. They appear, when the Fermi level passes through the singularity point.
\end{abstract}

